\title{
Theories of Elasticity with Couple-stress
}

\author{
R. A. ToupIN
}

\section{Introduction}

The concept of couple-stress is familiar from the theory of elastic shells. It is customary to represent the action of one part of a shell upon another by a line distribution of forces and couples along a curve which divides the shell into two parts. If suitable other assumptions be made, the force per unit length $_{(n)} \boldsymbol{t}$, and the couple per unit length ${ }_{(n)} \mathfrak{m}$ at a point $P$ of the shell which act on the curve through $P$ with normal $n$ are given by

$$
(n)^{t^{i}}=t^{i j}(P) n_{j}, \quad(n)^{\mathfrak{m}^{i}}=\mathfrak{m}^{i j}(P) n_{j},
$$

where $t^{i j}(P)$ is the stress tensor at $P, \mathfrak{m}^{i j}(P)$ is the couple-stress tensor at $P$, and $n_{j}$ is the normal to the curve which lies in the shell. The stress and couplestress at a given point $P$ are determined by the local instantaneous configuration of the material points in an arbitrarily small neighborhood of $P$. One problem of shell theory is to deduce these stress and couple-stress-deformation relations for a two-dimensional medium (i.e., a shell represented mathematically by a surface) from given stress-deformation relations of an elastic three-dimensional medium of which the shell is regarded as a thin piece.

In the classical theory of three-dimensional elastic media, the action of one part of a body upon another is represented by a distribution of forces only upon the dividing surface, and the stress vector at a point $P$ of a surface with normal $n_{i}$ is again given by $(1.1)_{1}$. The whole of the classical theory of elasticity seems consistent with the assumption that the couple-stress vanishes. Our purpose here will be to review several mathematical models of elastic continua to which this central feature of the classical theories of deformable media fails of extension. Besides a general consideration of these models which goes deeply into the basic principles of continuum mechanics, we shall show by examples the sort of phenomena embraced by these more elaborate models of elastic media and excluded by the simpler classical model. While these applications are now few and represent delicate refinements of the classical theory, they might easily grow in number and diversity. At the present time, however, perhaps the best recommendation for study of these particular models is the thoughtful re-examination of the basic concepts and principles of continuum mechanics they require for understanding. They allow one to view the classical theory itself in new perspective.

The following brief summary of the literature on couple-stresses begins appropriately with the memoir of E. \& F. Cosserat $(1909,1)$. The Cosserats 
gave a systematic development of the mechanics of continuous media each point of which has the six degrees of freedom of a rigid body. In classical elasticity theory, a material point has only the three degrees of freedom corresponding to its position in Euclidean space. They were drawn to the general concept of such a medium by various special theories of elastic rods and shells, and they extended the notion in a natural way to three-dimensional media. The most striking and novel feature of their theory of three-dimensional media was the appearance of couple-stresses in the equations of motion. The stress tensor in the Cosserat theory was not symmetric as in the classical theory of elasticity but satisfied rather the equation*

$$
t^{[i j]}+m^{i i k}=0
$$

jointly with the couple-stress tensor field when the body is in equilibrium. While the counterpart of (1.2) was known for shells, and the idea of couple-stress for three-dimensional media had occurred to VoIGT and others, the CossERATs were the first to treat systematically the mechanics of an elastic medium in which couple-stresses were a central consideration. Although their work is a landmark in the development of continuum mechanics, it did not receive much comment or search for applications. HELLINGER and von HEUN drew attention to their ideas (1914, 1 and 2), and TRUeSDELL remarked upon equation (1.2) in his article, "The Mechanical Foundations of Elasticity and Fluid Mechanics" (1952, 1). ERICKSEN \& TRUESDELL $(1958,1)$ developed further the purely kinematical description of Cosserat continua emphasizing the one- and two-dimensional cases of rods and shells; but they did not explore or amplify the theory of motion of elastic media proposed by the Cosserats. Also in $(1958,2)$, GüNTHER pointed out the connection between the kinematics of a Cosserat continuum and the new theories of continuous distributions of dislocations then emerging. $\mathrm{H}$. ScHAEFER $(1962,3)$ solved some explicit boundary value problems for a twodimensional Cosserat medium so as to illustrate some of the novel features of the theory.

JARAMILlo $(1929,1)$ constructed a different but related generalization of the classical theory of infinitesimal elastic deformations based on the assumption that the action density in Hamilton's principle was a quadratic function of the second- order spatial derivatives of the displacement field as well as the firstorder spatial derivatives and velocity components which appear in the action density of the classical theory. Relying too strongly on the classical stress principle and certain of its consequences such as the symmetry of the stress tensor, JARAMILlo was led to impose unnatural restrictions on the dependence of the action density upon the second-order spatial derivatives of the displacement field. In any case, he recognized that such a medium would be dispersive; i.e., that the speed of a sound wave would depend on its frequency. Tifren $\&$ Stevenson $(1955,1)$ also considered briefly the theory of infinitesimal motions

* Square brackets about any set of indices denotes the average of the alternating sum over the permutations of the indices; e.g., $t^{[i j]}=\frac{1}{2}\left(t^{i j}-t^{i j}\right)$. The couple-stress field can be represented alternatively by a second rank axial tensor field $\mathrm{m}^{i j}$ as in (1.1) or by the dual absolute third rank tensor $m^{i j k}$. The relation between $\mathfrak{m}^{i j}$ and its dual $m^{i j k}$ is $m^{i j k}=\frac{1}{2} e^{i j} \mathrm{~m}^{s k}$, where $e^{i j k}$ is the axial alternating tensor which has components $1,-1$, or 0 in Cartesian frames of reference. 
of such an elastic medium of "grade 2 ". It is convenient to call a material of "grade $N$ " if $N$ is the order of the highest position gradient in the action density. Tiffen \& Stevenson saw that couple-stresses exist in such media and rediscovered the CosSERATs' equation (1.2) for the balance of moments. It turns out, as will be shown later, that if the rotation of a point in a Cosserat continuum is constrained to equal the local rotation of the medium in the familiar sense of elasticity and fluid dynamics, the theory which results is equivalent to a certain special case of the general theory of elastic materials of grade 2 . For ease of reference to this particular theory and set of ideas, let us call it the Cosserat theory with constrained rotations. Because of the constraints, in this theory the couple-stress tensor is not completely determined by the action density as it is in the Cosserats' original theory. This point was overlooked by Tiffen \& Stevenson and also by Truesdell \& Toupin $(1960,1)$ in their treatment of stress and couple-stress in materials of grade 2. GRIOLI $(1960,2)$ gave the first general and correct treatment of elastic materials of grade 2 whose energy equation was of the same form as the CossERATS' energy equation; i.e., of Cosserat media with constrained rotations. In $(1962,1)$, I reviewed the foundations of the theory of elastic materials of grade 2 , corrected the formula for the couple-stress given in $(1960,1)$, and pointed out that the Cosserat media with constrained rotations were but a peculiar subclass of the elastic materials of grade 2. At the same time, Mindlin \& Tiersten $(1962,2)$ gave an extensive analysis of infinitesimal motions of Cosserat media with constrained rotations. They extended to this more general theory of elasticity many of the classical results on stress functions, fundamental solutions, vibrations, nuclei of strain, etc. Also, explicit solutions of some boundary value problems were constructed which illustrated the novel departures from classical results predicted by the theory. ToupIN \& GAZIS $(1963,1)$ applied the general theory of materials of grade 2 to the problem of surface deformations of a crystal. They showed that initial stress and "hyperstress" in a uniform crystal gave rise to a deformation of a thin boundary layer near a free surface such as had been observed in electron diffraction experiments.

In $(1958,1)$, ERICKSEN \& TRUESDELL suggested a natural generalization of Cosserat media. As remarked above, a Cosserat medium is a continuum, each point of which has the degrees of freedom of a rigid body. The orientation of a given point of such a medium can be represented mathematically by the values of three mutually perpendicular unit vectors which ERICKSEN \& TRUESDELL called the "directors" of an "oriented medium". With this idea in mind, it is easy and natural to consider the generalization in which the three directors are stretchable and not constrained to remain mutually orthogonal. Mindin $(1964,1)$ has now considered in some detail the mechanics of elastic media with "microstructure". The kinematical model of an oriented medium with deformable directors is arrived at by the following slightly different train of reasoning. Minduin begins with the very general concept of an elastic continuum each point of which is in itself a deformable medium. This concept stems from the easier notion that a rod or a shell may trivially be viewed as a one-dimensional manifold of two-dimensional deformable "points", or a two-dimensional manifold of one-dimensional deformable "points", respectively. If each "micromedium" 
is constrained to deform homogeneously, such a model reverts to ERICKSEN \& TRUESDELL's oriented medium with deformable directors because a homogeneous deformation is uniquely determined by the motion of any three linearly independent vectors (the directors).

In all the foregoing models of continuous media, couple-stresses occur in the analysis of their mechanical behavior, and the familiar concepts of stress which suffice for an understanding of classical elasticity theory prove inadequate. In the following sections, each of the models will be considered in greater or less detail. Our objective has been to expose the concepts and principles of continuum mechanics common to all the models and to devise a mathematical machinery for easy and precise expression of the basic ideas.

\section{Kinematical Preliminaries}

The Cosserats gave a systematic treatment of elastic curves, surfaces, and extended bodies. Each variety of continuum has its peculiar features and mathematics, but here we shall consider only the three-dimensional case.

Let $M$ be a three-dimensional manifold of material points which we denote by bold face Greek letters $\xi$, $\eta$, etc. Let $(\alpha),(\beta), \ldots$ denote the coordinate systems of $M$ and $\xi^{\alpha}(\xi), \alpha=1,2,3$, the material coordinates of $\xi$ in the coordinate system $(\alpha)$.

A motion of $M$ is a one-parameter family of mappings

$$
x_{t}: M \rightarrow E
$$

of $M$ into Euclidean space $E$. Coordinate systems of $E$ will be denoted by (i), $\left(i^{\prime}\right)$, etc. The image $\boldsymbol{x}=\boldsymbol{x}_{\boldsymbol{t}}(\xi)$ of $\boldsymbol{\xi}$ is called the position of $\boldsymbol{\xi}$ at the time $t$. More generally, the image $\boldsymbol{x}_{t}(S)$ of any set of points $S<M$ is called the configuration of $S$ at the time $t$. When coordinates are introduced in $M$ and $E$, a motion of $M$ becomes represented, at least locally, by three real-valued functions

$$
x^{i}=x^{i}\left(\xi^{\alpha}, t\right)
$$

of the four real variables $\xi^{\alpha}, \alpha=1,2,3$ and the time $t$.

The velocity of $\xi$ has the components

$$
x^{i}=\frac{\partial x^{i}\left(\xi^{\alpha}, t\right)}{\partial t},
$$

and the acceleration of $\xi$ has the components

$$
\ddot{x}^{i}=\frac{\partial \dot{x}^{i}\left(\xi^{\alpha}, t\right)}{\partial t}+\left\{\begin{array}{c}
i \\
j k
\end{array}\right\}_{g} \dot{x}^{j} \dot{x}^{k},
$$

where $\left\{\begin{array}{c}i \\ j k\end{array}\right\}_{g}$ are the Christoffel symbols computed from the components of the spatial metric tensor $g_{i j}\left(x^{k}\right)$. Throughout we assume that the spatial coordinate system is inertial and nondeforming so that the $g_{i j}\left(x^{k}\right)$ never depend on the time $t$. The spatial metric tensor reduces to the Kronecker delta $\delta_{i j}$ when the spatial coordinates are rectangular Cartesian.

We consider only motions for which the position gradient

has rank three throughout $M$.

$$
x_{, \alpha}^{i}=\frac{\partial x^{i}\left(\xi^{\alpha}, t\right)}{\partial \xi^{\alpha}}
$$


At each instant of time, a motion of $M$ induces in it a Euclidean metric field defined by

$$
a_{\alpha \beta}\left(\xi^{\alpha}, t\right)=g_{i j} x^{i}, \alpha x_{, \beta}^{j} .
$$

The instantaneous length, area, and volume of material curves, surfaces, and regions, respectively, are defined in the usual way in terms of the instantaneous material metric tensor $a_{\alpha \beta}\left(\xi^{\alpha}, t\right)$.

Let $C_{t}=x_{t}(M)$ denote the configuration of the body at time $t$. We shall need the concept of a reference configuration $C_{T}$ which, in the applications, will have special properties. We call $T$ the reference time and introduce the special notations

$$
\boldsymbol{X}=\boldsymbol{x}_{T}(\xi), \quad X^{i}=x^{i}\left(\xi^{\alpha}, T\right), \quad A_{\alpha \beta}\left(\xi^{\alpha}\right)=g_{i j} X_{, \alpha}{ }_{, \alpha} X^{j}, \beta
$$

for the position, coordinates of position, and material metric tensor at $\xi$ at the reference time. Since the mapping $X^{i}=x^{i}\left(\xi^{\alpha}, T\right)$ is one-to-one and invertible, it is always possible to introduce the $X^{i}(\xi)$ as material coordinates, but we wish not to restrict the formalism to this agreement.

Absolute differentiation, which requires a definition of parallelism in $M$, can be defined using the Christoffel symbols $\left\{\begin{array}{c}\alpha \\ \beta \gamma\end{array}\right\}_{a}$ of the instantaneous metric $a_{\alpha \beta}$. Alternatively, absolute differentiation of tensor fields in $M$ can be defined using the Christoffel symbols $\left\{\begin{array}{c}\alpha \\ \beta \gamma\end{array}\right\}_{A}$ of the fixed (time independent) values of the material metric tensor at the reference time $T$.

The gradient of the position gradient, or alternatively, the second-order position gradient will be here defined by

$$
x_{; \alpha \beta}^{i}=\frac{\partial^{2} x^{i}}{\partial \xi^{\alpha} \partial \xi^{\beta}}-\left\{\begin{array}{c}
\gamma \\
\alpha \beta
\end{array}\right\}_{A} x^{i}, \gamma+\left\{\begin{array}{c}
i \\
j k
\end{array}\right\}_{g} x^{j}, \alpha x_{, \beta}^{k} .
$$

This tensor is distinct, in general, from the tensor

$$
x_{\mid \alpha \beta}^{i}=\frac{\partial^{2} x^{i}}{\partial \xi^{\alpha} \partial \xi^{\beta}}-\left\{\begin{array}{c}
\gamma \\
\alpha \beta
\end{array}\right\}_{a} x^{i}, \gamma+\left\{\begin{array}{c}
i \\
j k
\end{array}\right\}_{\varepsilon} x^{j}, \alpha x_{, \beta}^{k} .
$$

The former of these two tensors, (2.8), reduces to the ordinary partial derivatives $\partial^{2} x^{i} \partial \xi^{\alpha} \partial \xi^{\beta}$ when the spatial coordinate system (i) is rectilinear and the material coordinate system $(\alpha)$ is such that the $A_{\alpha \beta}$ are constants.

\section{Continuous Media with Microstructure}

A rod or a shell can be viewed as the Cartesian product $M^{\mathbf{1}} \times M^{\mathbf{2}}$ of a onedimensional continuum $M^{\mathbf{1}}$ and a two-dimensional continuum $M^{2}$. For example, a rod may be regarded as the Cartesian product of its line of centroids in some configuration and of its cross section in the same configuration. Nothing is gained by such a formal step. Theories of rods and shells gain simplicity over a straightforward representation as three-dimensional bodies by restricting the deformations of one of the factors in the product $M^{3}=M^{1} \times M^{2}$. One can, for example, constrain the cross sections of a rod or the lines normal to the midsection of a shell to undergo only homogeneous deformations. But a homogeneous deformation of an $n$-dimensional continuum is uniquely determined by the 
motion of any $n$ linearly independent vectors joining one of its points to $n$ others. In this way, one arrives at the kinematical model of a rod consisting of a onedimensional continuum $M^{1}$ and a set of two vector fields $\left(d_{1}^{i}(\xi, t), d_{2}^{i}(\xi, t)\right)$ in $M^{1}$ whose values fix a homogeneous deformation of the cross section of the rod through the point $\xi$. In the same spirit, one could represent a shell as a twodimensional continuum $M^{2}$ and a single vector field $d^{i}(\xi, t)$ whose values determine a homogeneous deformation of a material line through $\xi$. More generally now, in light of these examples, it is easy to conceive a curve, surface, or region, each point of which is an $n$-dimensional continuum constrained to deform homogeneously. Such a model would be represented mathematically by a one-, two-, or three-dimensional manifold $M$ and a set of $n$ spatial vector fields $d_{\mathbf{a}}^{i}(\xi, t)$, $\mathfrak{a}=1,2, \ldots, n$. If in each case one chooses $n=3$ and further assumes that each "micromedium" moves rigidly, then

$$
g_{i j} \underset{\mathfrak{a}}{d_{\mathfrak{b}}^{i}} d^{j}=\underset{\mathfrak{a} \mathfrak{b}}{\delta}, \quad \mathfrak{a}, \mathfrak{b}=1,2, \mathfrak{3},
$$

and the resulting model is a Cosserat continuum. Following ERICKSEN \& TruesDELL, we call the vector fields $\underset{a}{d}(\xi, t)$ directors. We shall consider the case of deformable directors for which the Cosserats' rigidity condition (3.1) is not imposed.

Rods and shells provide specific and practical examples of one- and twodimensional continua with one or more deformable directors. An electrically polarizable medium with its polarization field $P^{i}(\xi, t)$ may be cited as a familiar example of a three-dimensional continuum with one "director". The polarization field serves not only to define a charge distribution but ascribes also to each point of the continuum a certain structure. A continuum with director fields resembles also a polyatomic lattice. One may identify points $\xi$ with unit cells and the director $\underset{a}{d}(\xi, t)$ as the position vector of the atomic species " $a$ " relative to the center of mass or charge of the cell $\xi$. All that we shall have to say about the mechanics of such oriented continua will be more or less independent of specific physical interpretation of the director fields. Also, it will be fairly obvious how to extend the basic results on oriented media to the more general model in which the vector fields $\underset{a}{d^{i}}(\xi, t)$ are replaced by any set of tensor fields $\varphi^{i j \ldots}(\xi, t)$ which might be introduced to describe the kinematical structure of a material point.

\section{Rigid Motions}

In classical elasticity theory, a motion of a body is rigid if and only if the rectangular Cartesian coordinates $x^{i}(\xi, t)$ of the position of every material point $\xi$ at time $t$ are given in terms of the rectangular Cartesian coordinates $X^{i}(\xi)$ of the reference positions by a relation of the form

$$
x^{i}(\xi, t)=R_{j}^{i}(t) X^{j}(\xi)+V^{i}(t)
$$

where $\boldsymbol{R}(t)$ is a proper orthogonal matrix and $\boldsymbol{V}$ is some vector-valued function of the time. For a medium with deformable directors we amend this definition 
of rigid motion by adding the conditions

$$
\underset{\mathfrak{a}}{d^{i}}(\xi, t)=R_{j}^{i}(t) \underset{a}{D^{j}}(\xi)
$$

where $\boldsymbol{R}(t)$ is the same matrix that appears in (4.1) and $\underset{a}{D^{i}}=\underset{a}{d^{i}}(\xi, T)$ are the values of the director fields at the reference time.

It follows from (4.1) and (4.2) that necessary conditions for rigid motion are

$$
a_{\alpha \beta}(\xi, t)=A_{\alpha \beta}(\xi), \quad \underset{\mathfrak{a b}}{q}(\xi, t)=\underset{a \mathfrak{b}}{Q}(\xi),
$$

where $a_{\alpha \beta}$ and $A_{\alpha \beta}$ are the metric components defined in (2.6) and (2.7) and

$$
\underset{\mathfrak{a} \mathfrak{b}}{q(\xi}, t)=g_{i j} d_{\mathfrak{a} b}^{i} d^{j}, \quad \underset{\mathfrak{a} \mathfrak{b}}{Q}(\xi, t)=\underset{\mathfrak{a} \mathfrak{b}}{q}(\xi, T)
$$

For the three-dimensional media under consideration here, the position gradient $x^{i}{ }_{, \alpha}$ is nonsingular. If it also be assumed that the matrix of components $D_{\mathfrak{a}}^{i}$ is nonsingular (i.e., that the initial values of the directors are linearly independent), then it can be shown that the conditions (4.3) are sufficient that a motion be rigid. If the conditions (4.3) hold at every point $\xi$ in some neighborhood $N(\xi)$ of $\xi$, then the motion of that neighborhood is rigid.

Other necessary conditions that a motion be rigid may be derived from (4.1) and (4.2) by differentiation; e.g.,

$$
\begin{aligned}
& x^{i}{ }_{, \alpha}(\xi, t)=R_{j}^{i}(t) X^{i}{ }_{, \alpha}(\xi), \\
& d_{a} d_{, \alpha}^{i}(\xi, t)=R_{j}^{i}(t) D_{a}{ }^{i}, \alpha(\xi) \text {. }
\end{aligned}
$$

\section{Hamilton's Principle for Perfectly Elastic Media}

Let $P<M$ be any part of an elastic medium with deformable directors, and let $I$ be an arbitrary time interval $t_{1} \leqq t \leqq t_{2}$. Following the CoSSERATS, we assign to every set of events $P \times I$ an amount of action $A(P \times I)$ given by

where

$$
A(P \times I)=\int_{I} \int_{P} L(\xi, t) d \mathscr{V} d t,
$$

$$
d \mathscr{V}=\sqrt{A} d \xi^{1} d \xi^{2} d \xi^{3}, \quad A=\operatorname{det}\left\|A_{\alpha \beta}\right\|
$$

is the differential element of volume in $P$ in the reference configuration, and $L(\xi, t)$ is the density of action. We assume that the action density is a function of the variables indicated now:

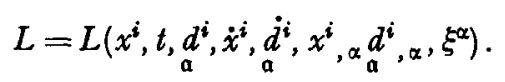

According to Hamilton's principle the equations of motion and boundary conditions for a material with action given by (5.1) are the Euler equations and the boundary conditions of the variational equation

$$
\begin{aligned}
& \delta A(P \times I)+\int_{I} \int_{P}\left(F_{i} \delta x^{i}+\stackrel{\mathfrak{a}}{G_{i}} \delta d_{\mathfrak{a}}^{i}\right) d \mathscr{V} d t+ \\
& +\int_{I} \int_{\partial P}\left(T_{i} \delta x^{i}+\stackrel{\mathfrak{H}}{H}_{i} \delta d_{\mathfrak{a}}^{i}\right) d \mathscr{A} d t-\left.\int_{P}\left(P_{i} \delta x^{i}+\stackrel{\mathfrak{Q}}{\mathrm{a}}_{i} \delta d_{\mathfrak{a}}^{i}\right) d \mathscr{V}\right|_{i_{1}} ^{t_{1}}=0
\end{aligned}
$$


where the $F_{i}$ and $\stackrel{a}{G}$ are certain generalized body forces, the $T_{i}$ and $\stackrel{a}{H}_{i}$ are certain generalized surface tractions, and the $P_{i}$ and $\stackrel{a}{Q}_{i}$ are certain generalized momenta.

The variational equation (5.4) is postulated for arbitrary differentiable variations $\delta x^{i}$ and $\delta d_{a}^{i}$ of the independent field variables. Necessary and sufficient conditions that (5.4) be satisfied for this class of variations are the Euler equations

where

$$
\begin{aligned}
\dot{P}_{i}^{*}-T_{i, \alpha}^{\alpha}-F_{i} & =\frac{\partial L}{\partial x^{i}}, \\
\stackrel{a}{Q_{i}^{*}}-\stackrel{a}{H}_{i, \alpha}^{\alpha}+\stackrel{a}{G}_{i}^{*}-\stackrel{a}{G}_{i} & =0,
\end{aligned}
$$

$$
P_{i}^{*}=\frac{\partial L}{\partial \dot{x}^{i}}, \quad T_{i}^{\alpha}=-\frac{\partial L}{\partial x_{, \alpha}^{i}}, \quad \stackrel{a}{Q}{ }_{i}^{*}=\frac{\partial L}{\partial \dot{d}^{i}}, \quad \stackrel{a}{H_{i}^{\alpha}}=-\frac{\partial L}{\partial d_{a}^{i}, \alpha}, \quad \stackrel{a}{G_{i}^{*}}=-\frac{\partial L}{\partial d_{a}^{i}},
$$

and the boundary conditions

$$
\begin{aligned}
& T_{i}^{\alpha} N_{\alpha}-T_{i}=0 \\
& \stackrel{a}{H_{i}^{\alpha}} N_{\alpha}-\stackrel{\mathfrak{a}}{H}_{i}=0, \quad \xi \in \partial P, \quad t \in I
\end{aligned}
$$

where $N_{\alpha}$ is the unit normal to $\partial P\left(A^{\alpha \beta} N_{\alpha} N_{\beta}=1\right)$, and the following conditions on the initial and final momenta

$$
\begin{aligned}
& P_{i}^{*}-P_{i}=0, \quad \xi \in P, \quad t=t_{1} \text { or } t_{2}, \\
& \stackrel{a}{Q}-\stackrel{a}{Q}=0 .
\end{aligned}
$$

When $L$ is independent of $x^{i}, t, \underset{a}{d}, \underset{a}{d_{a}}$, and $\underset{a}{d^{\prime} \alpha}$ and has the special form

$$
L=\frac{1}{2} \varrho\left(\xi^{\alpha}\right) \dot{x}^{2}-W\left(x_{, \alpha}^{i}, \xi^{\alpha}\right),
$$

the equations $(5.7)_{1}$ and $(5.5)_{1}$ reduce to the equations of motion and traction boundary conditions of the classical theory of finite elastic deformations. The tensor $T_{i}^{\alpha}$ in this special case is the Kirchhoff stress tensor. Cauchy's stress tensor in the classical theory is defined by

$$
t^{i j}=\sqrt{\frac{A}{a}} T^{i \alpha} x_{, \alpha}^{j}
$$

and (in the classical theory) is symmetric if and only if the energy of deformation $W\left(x_{, \alpha}^{i}, \xi^{\alpha}\right)$ is invariant under rigid rotations. We proceed now to show how these familiar results of the classical theory are modified in elastic media with deformable directors.

\section{Invariance and Conservation}

We shall say that two motions of a given medium differ by a Euclidean displacement if

$$
\begin{aligned}
x^{* i}\left(\xi, t^{*}\right) & =R_{j}^{i} x^{i}(\xi, t)+D^{i}, \\
d_{\mathfrak{a}}^{* i}\left(\xi, t^{*}\right) & =R_{j}^{i} d_{\mathfrak{a}}^{i}(\xi, t), \\
t^{*} & =t+C,
\end{aligned}
$$

where the starred quantities define one of the motions and the unstarred quantities the other. All quantities are referred to a common rectangular Cartesian inertial frame of reference, $R_{j}^{i}$ is a constant proper orthogonal matrix, and the $D^{i}$ and $C$ are also constants. The set of all transformations (6.1) of the independent 
field quantities has the group property. We call it the group of Euclidean displacements. Two motions of a given medium related by an element of the group as in (6.1) are said to be congruent.

Following the Cosserats, we postulate that the action density $L$ is invariant under the group of Euclidean displacements. In other words, the action density has the same value for all congruent motions.

Since the group of Euclidean displacements is a connected Lie group, it is sufficient to require that the action density be invariant under infinitesimal transformations of the group of Euclidean displacements in order that it be invariant under arbitrary, finite transformations of the group. An infinitesimal transformation of the group has the form

$$
\begin{array}{rl}
x^{* i} & =x^{i}+\left(\Omega^{i j} x_{j}+D^{i}\right) d \lambda, \\
d^{* i} & =d^{i}+\Omega^{i j} d_{j} d \lambda, \\
a & a \\
t^{*} & =t+C d \lambda,
\end{array}
$$

where, except for the antisymmetry condition $\Omega^{i j}=-\Omega^{j i}$, the $\Omega^{i j}, D^{i}$, and $C$ are arbitrary constants. It is easy now to deduce that the action density will be invariant under the group of Euclidean displacements if and only if

where

$$
\frac{\partial L}{\partial x^{i}}=\frac{\partial L}{\partial t}=0, \quad K_{[i j]}=0
$$

$$
K_{i j}=x_{i} \frac{\partial L}{\partial x^{j}}+d_{\mathfrak{a}} \frac{\partial L}{\partial d_{\mathfrak{a}}^{j}}+\dot{x}_{i} \frac{\partial L}{\partial \dot{x}^{j}}+\dot{d}_{\mathfrak{a}} \frac{\partial L}{\partial \dot{d}_{\mathfrak{a}}^{j}}+x_{i, \alpha} \frac{\partial L}{\partial x_{, \alpha}^{j}}+d_{\mathfrak{a}, \alpha} \frac{\partial L}{\partial d_{\mathfrak{a}, \alpha}^{j}} .
$$

With the conditions (6.3) in mind, consider now the variational equation (5.4) which summarizes the equations of motion, boundary conditions, and initial and final data. We know that, in particular, (5.4) must hold for all variations of the special varieties,

$$
\begin{aligned}
& \text { (1) }\left(\delta x^{i}=D^{i}, \quad \delta d_{a}^{i}=0\right) \text {, } \\
& \text { (2) } \quad\left(\delta x^{i}=\Omega^{i j} x_{j}, \quad \delta \underset{\mathfrak{a}}{d^{i}}=\Omega^{i j}{ }_{\mathfrak{a}}{ }_{j}\right) \text {, } \\
& \text { (3) }\left(\delta x^{i}=\dot{x}^{i}, \quad \delta \underset{\mathbf{a}}{d^{i}}=\dot{d}_{\mathfrak{a}}^{i}\right) \text {, }
\end{aligned}
$$

since we postulate (5.4) for perfectly arbitrary smooth variations. Necessary and sufficient conditions that (5.4) hold for these special types of variations are

$$
\begin{aligned}
& \int_{P} P_{i} d \mathscr{V} \int_{t_{1}}^{t_{2}}-\int_{I} \int_{P} F_{i} d \mathscr{V} d t-\int_{I} \int_{P} T_{i} d \mathscr{A} d t=\int_{I} \int_{P} \frac{\partial L}{\partial x^{i}} d \mathscr{V} d t, \\
& \left.\int_{P} S_{i j} d \mathscr{V}\right]_{t_{\mathfrak{I}}}^{t_{\mathfrak{z}}}-\int_{I} \int_{P}\left(x_{[i} F_{j]}+d_{\mathfrak{a}} \stackrel{\mathfrak{a}}{G}_{j]}\right) d \mathscr{V} d t-\int_{I} \int_{P}\left(x_{[i} T_{j]}+d_{\mathfrak{a}} \stackrel{\mathfrak{a}}{H}_{j]} d \mathscr{A} d t\right. \\
& =\int_{I} \int_{P} K_{[i j]} d \mathscr{A} d t
\end{aligned}
$$

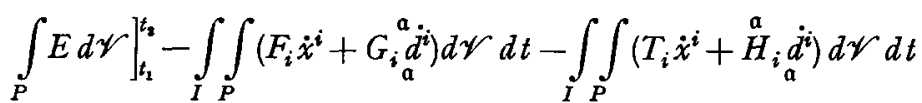

$$
\begin{aligned}
& =\int_{I} \int_{P} \frac{\partial L}{\partial t} d \mathscr{V} d t
\end{aligned}
$$


where, in (6.7), we have set

$$
E=P_{i} \dot{x}^{i}+\stackrel{a}{Q_{i}} \dot{d}_{a}^{i}-L,
$$

which we call the energy density, and in (6.6) we have set

$$
S_{i j}=-S_{j i}=x_{[i} P_{j]}+d_{\mathfrak{a}} \stackrel{\mathfrak{a}}{Q}_{j]},
$$

which we call the density of angular momentum. Inspection of (6.5), (6.6) and (6.7) shows that if the right-hand sides were to vanish, then the difference between the linear momentum, angular momentum, and energy of the set of points $P$ at times $t_{2}$ and $t_{1}$ would equal, respectively, the resultant linear impulse, the resultant angular impulse, and the total work done by the generalized forces $F_{i}, \stackrel{a}{G}_{i}, T_{i}$, and $\stackrel{a}{H}_{i}$ during the interval of time $l$. This is a common way of expressing the laws of conservation of linear momentum, angular momentum, and energy. Now the right-hand sides of (6.5), (6.6), and (6.7) vanish for every set of particles $P$ and for every interval of time $I$ if and only if the action density is invariant under the group of Euclidean displacements, as can easily be seen from (6.3). Thus, we have established the basic theorem of equivalence between conservation and invariance:

Linear momentum, angular momentum, and energy are conserved in a perfectly elastic medium with deformable directors if and only if the action density is invariant under the group of Euclidean displacements.

From the definition (6.9) of the density of angular momentum one sees that, in general, the angular momentum of a particle does not equal the moment of its linear momentum as in classical elasticity theory and fluid dynamics. Also, from (6.5) and (6.6) one sees that the torque exerted on a body is not, in general, equal to the moment of the body forces $F_{i}$ and surface tractions $T_{i}$ but is greater by an amount equal to the volume integral of $\underset{\mathfrak{a}}{\boldsymbol{d}} \times \stackrel{\mathfrak{G}}{\boldsymbol{G}}$ plus the surface integral of $\underset{\mathfrak{a}}{\boldsymbol{d}} \times \stackrel{\mathfrak{a}}{\boldsymbol{H}}$. It follows that these quantities represent volume and surface distributions of couples, respectively.

That the conservation of energy, momentum, and angular momentum are necessary and sufficient conditions for the Euclidean invariance of the action density in Hamilton's principle was emphasized again and again in the CossERATs' memoir. In this section we have merely summarized and adapted their results for three-dimensional media given in $(1909,1, \S \S 64-65)$. The general theory of the connection between invariance and conservation in the variational calculus is generally attributed to F. KLeIN $(1918,1)$ and E. Noether $(1918,2)$. The counterparts of $(6.5),(6.6)$, and $(6.7)$ for the general elastic material of grade 2 were presented in $(1963,3)$.

\section{Stress, Hyperstress, and Couple-Stress}

To cast the equations of motion and boundary conditions (5.5) and (5.7) into a more familiar form let us extend the classical definition of Cauchy's stress tensor to the present case and set

$$
t_{i}^{j}=j^{-1} T_{i}^{\alpha} x_{, \alpha}^{j}=-j^{-1} \frac{\partial L}{\partial x^{i}, \alpha} x^{j}, \alpha
$$


Alongside Cauchy's stress tensor let us set the hyperstress tensor, defined by

$$
h_{i j}{ }^{k}=-j^{-1} d_{\mathbf{a}} \frac{\partial L}{\partial d_{\mathfrak{a}}, \alpha} x^{k}, \alpha
$$

where $j$ is the absolute scalar defined by

$$
j=\sqrt{\frac{a}{A}} .
$$

Let us also introduce the generalized forces and momenta per unit deformed (present) volume and area defined by

$$
\begin{aligned}
& f_{i}=j^{-1} F_{i}, \quad t_{i}=T_{i} \frac{d A}{d a}, \quad l_{i j}=j^{-1} \underset{\mathbf{a}}{d_{i}} \stackrel{a}{G_{j}}, \\
& p_{i}=j^{-1} I_{i}, \quad h_{i j}={\underset{a}{a}}_{i} \stackrel{\mathfrak{a}}{H}_{j} \frac{d A}{d a}, \quad j q_{i j}=Q_{i j}=d_{\mathfrak{a}} \stackrel{\mathfrak{a}}{Q}_{j} .
\end{aligned}
$$

The antisymmetric part of $l_{i j}$ is a certain couple per unit deformed volume, the antisymmetric part of $h_{i j}$ is a certain couple per unit deformed area, and the antisymmetric part of $q_{i j}$ is a certain spin angular momentum per unit deformed volume.

In terms of the above quantities, which have an easy interpretation, the equations of motion and boundary conditions (5.5) and (5.7) may be expressed as follows:

$$
\begin{aligned}
j^{-1} \dot{P}_{i} & =t_{i, j}^{j}+f_{i}, \\
j^{-1} \dot{Q}_{i j} & =h_{i j, k}{ }^{k}+t_{j i}+l_{i j}+j^{-1}\left(K_{i j}-\dot{x}_{i} P_{j}\right), \\
t_{i}{ }^{j} n_{j} & =t_{i}, \\
h_{i j}{ }^{k} n_{k} & =h_{i j},
\end{aligned}
$$

where $n_{i}$ is the unit outward normal to the deformed configuration of the body.

When the action density is of such a form that

$$
P_{i}=\frac{\partial L}{\partial \dot{x}^{i}}={ }_{0} \varrho \dot{x}_{i}
$$

and $\dot{\varrho}=0$, then Eq. (7.5) becomes

$$
\varrho \ddot{x}_{i}=t_{i, j}^{j}+t_{i},
$$

and the antisymmetric part of Eq. (7.6) becomes

$$
\varrho \dot{s}_{i j}=m_{i j, k}^{k}+t_{[j i]}+l_{[i j]},
$$

where $\varrho=j^{-1}{ }_{0} \varrho$ is the mass per unit deformed volume,

$$
s_{i j}=\frac{1}{\varrho} q_{[i j]}
$$

is the spin angular momentum per unit mass, and

$$
m_{i j}^{k}=h_{[i j]^{k}}
$$


is the couple-stress tensor. Eqs. (7.10) resemble formally CAUCHY's equations of motion for a continuous medium, and Eqs. (7.11) are the CossERATs' equations of motion for the spin. If the action density be independent of the directors, their time derivatives and their gradients, then (7.11) reduces to the familiar result,

in the absence of body couples.

$$
t_{i j}=t_{j i}
$$

The symmetric part of equation (7.6) is not so easy to interpret in terms of familiar concepts. However, when the action density is of such a form that

then

$$
\frac{\partial L}{\partial \dot{d}_{\mathfrak{a}}^{i}}=\stackrel{\mathfrak{a}}{Q_{i}}=\stackrel{\mathfrak{a} \mathfrak{v}}{\mathfrak{b}} \dot{d}_{\mathfrak{b}}, \quad \stackrel{\mathfrak{a} \dot{b}}{\mathfrak{v}}=0
$$

$$
Q_{(i j)}=\frac{1}{2} \underset{v}{\nu} \underset{a}{d_{(i b} d_{j)}}=\dot{c}_{i j}
$$

is the time rate of change of the form factor $c_{i j}(\xi, t)$ of the particle $\xi$. Especially, if one returns to the interpretation of the directors as defining a homogeneous deformation of a particle, one sees that $c_{i j}$ is a certain measure of strain of a particle. The symmetric part of Eq. (7.6) then gives an equation for the acceleration of the microstraining.

\section{Measures of Strain, Microstrain, and Relative Strain}

Let us assume for simplicity that the action density satisfies the two conditions (7.9) and (7.15), which insure that the linear momentum is parallel to the velocity of the medium and that the momenta conjugate to the directors are linear functions of the time rates of change of the directors. These conditions are necessary and sufficient that the action density have the form

$$
L=T-W
$$

where

$$
T=\frac{1}{2}\left(\varrho g_{i j} \dot{x}^{i} \dot{x}^{j}+g_{i j} \underset{\mathfrak{a}}{\stackrel{a}{v} \underset{d^{i}}{i}} \dot{d}^{j}\right)
$$

is the kinetic action (kinetic energy), and the negative of

$$
W=W\left(x^{i}, t, d_{\mathfrak{a}}^{i}, x^{i},{ }_{\mathfrak{a}}, d^{i}, \xi^{\nu}\right)
$$

is the action of deformation ( $W$ is the energy of deformation) which is independent of $\dot{x}^{i}$ and $\dot{d}_{\mathfrak{a}}^{i}$. In this case, the energy density, as defined in (6.8), has the familiar form

$$
E=T+W .
$$

Now the kinetic part of the action, as given in (8.2), is invariant under Euclidean displacements; hence, the energy of deformation must be separately invariant under Euclidean displacements. An immediate consequence is that $W$ must be independent of $x^{i}$ and $t$, and we can write

$$
W=W\left(d_{a}^{i}, x^{i}, \alpha, d_{a}^{i}, \alpha, \xi^{\alpha}\right) .
$$


Under the group of Euclidean displacements the material coordinates $\xi^{\alpha}(\xi)$ of a point are invariant and each of the other quantities which appear as arguments of $W$ transforms as a vector for each fixed value of $a$ and $\alpha$. In other words, every admissible energy function $W$ must satisfy the functional equation

$$
W\left(R_{j}^{i}{ }_{\mathfrak{a}}^{d^{j}}, R_{j}^{i}{ }_{j}^{j}, \alpha, R^{i}{ }_{\mathfrak{a}} d^{j}, \alpha, \xi^{\alpha}\right)=W\left(d_{\mathfrak{a}}^{i}, x^{i}, \alpha, d_{\mathfrak{a}}^{i}, \alpha, \xi^{\alpha}\right)
$$

for all values of the arguments in the domain of $W$ and for all proper orthogonal matrices $R^{i}{ }_{j}$. CAUCHY initiated the theory of such invariant functions of vectors, and he showed $(1850,1)$ that the general solution of the functional equation (8.6) was an arbitrary function of the inner products of the vector arguments taken two at a time and of the determinants of the vector arguments taken three at a time, plus, of course, in our case, an arbitrary dependence on the material coordinates $\xi^{\alpha}(\xi)$ of the point in question. In addition to the $\xi^{\alpha}$, there is a total of fifteen vector arguments of $W$ listed in (8.5), and the $\left(\begin{array}{c}15 \\ 2\end{array}\right)+15$ inner products plus the $\left(\begin{array}{c}15 \\ 3\end{array}\right)$ determinants are not all functionally independent. We wish to prove that, when the domain of the energy function $W$ is restricted by the condition

$$
\operatorname{det}\left\|x^{i}, \alpha\right\| \neq \text {, }
$$

every solution of (8.6) is expressible as a function of the smaller number of invariants

$$
\begin{aligned}
a_{\alpha \beta} & =g_{i j} x_{, \alpha}^{i} x^{j}, \beta \\
\Delta_{\alpha} & =g_{i j} x^{i},{ }_{a}^{d^{j}}, \\
\Delta_{\alpha \beta} & =g_{i j} x^{i},{ }_{a}^{d^{j}, \beta}, \\
s & =\operatorname{sgn} \operatorname{det}\left\|x^{i}, \alpha\right\| \text { and } \xi^{\alpha}(\xi) .
\end{aligned}
$$

Proof. Throughout the restricted domain (8.7), $x^{i}{ }_{\alpha}$ is nonsingular and (8.8) ${ }_{2}$, $(8.8)_{3}$ can be solved uniquely for $d_{a}^{i}$ and $\underset{\alpha}{d_{\alpha}, \alpha}$ in terms of $x^{i}, \alpha, \Delta_{\alpha \beta}$, and $\Delta_{\alpha}$. Thus, $W=W\left(x^{i}, \alpha, \Delta_{\alpha}, \Delta_{a \beta}, \xi^{\alpha}\right)$. Using Cauchy's theorem and the identity

$$
g\left(\operatorname{det}\left\|x^{i}, \alpha\right\|\right)^{2}=\operatorname{det}\left\|a_{\alpha \beta}\right\|
$$

which fixes the value of the determinant up to its sign in terms of the metric components, one sees that the assertion is true.

In elasticity theory, it is customary to introduce the coordinates $X^{\boldsymbol{i}}(\xi)$ of the material points $\xi$ in a reference configuration as independent variables in place of the material coordinates $\xi^{\alpha}$ and to describe the motion of $M$ by the mapping $x^{i}=\hat{x}^{i}\left(X^{1}, X^{2}, X^{3}, t\right)$. If this be done in the present case and one assumes that the energy $W$ is a function of the variables

$$
\begin{aligned}
x^{i}, \hat{j} & \equiv \frac{\partial \hat{x}^{i}}{\partial X^{j}}, \\
x^{i}, \hat{j} \hat{k} & \equiv \frac{\partial^{2} \hat{x}^{i}}{\partial X^{j} \partial X^{k}}+\left\{\begin{array}{c}
i \\
j k
\end{array}\right\}_{g(x)} x^{j}, \hat{j} x^{k}, \hat{k}-\left\{\begin{array}{c}
\hat{l} \\
\hat{j} \hat{k}
\end{array}\right\}_{g(X)} x^{i}, \hat{\imath}
\end{aligned}
$$


rather than the $x_{, \alpha}^{i}$ and $x^{i}, \alpha \beta$, then the foregoing results hold with obvious changes in notation and meaning of the variables. Moreover, if the reference and present configurations are referred to one and the same rectangular Cartesian coordinate system, then $\hat{s} \equiv \operatorname{det}\left\|\partial x^{i} / \partial X^{i}\right\|$ will always have a positive value since $\hat{s}(\xi, T)=1$ and $\hat{s}(\xi, t)$ is never zero. Thus, $\hat{s}$ may be eliminated as a variable in the energy function because the domain of $W$, in this case, is restricted to positive values of $\hat{s}$.

Returning now to the discussion of strain measures, consider the six independent quantities

$$
e_{\alpha \beta}=\frac{1}{2}\left(a_{\alpha \beta}-A_{\alpha \beta}\right) \text {. }
$$

Each of these quantities vanishes whenever the distance between every pair of neighboring material points is the same as in the reference configuration. Thus, they serve as measures of the strain of the present configuration $x_{t}(M)$ relative to the reference configuration $x_{T}(M)$ of the body. When the rectangular Cartesian components of the displacement field

$$
u^{i}=x^{i}(\xi, t)-X^{i}(\xi)
$$

yield an infinitesimal displacement gradient

$$
u_{i: j}=\frac{\partial u_{i}}{\partial X^{j}}, \quad\left|u_{i: j}\right| \ll 1
$$

and the $X^{i}(\xi)$ are chosen for material coordinates $\xi^{\alpha}(\xi)$, then

$$
e_{i j} \cong \tilde{e}_{i j}=u_{(i: j)}=\frac{1}{2}\left(u_{i: j}+u_{j: i}\right)
$$

to first order terms in the $u_{i: j}$. The symmetric part $\tilde{e}_{i j}$ of the displacement gradient is the measure of relative strain in the classical linear theory of elasticity. The $e_{\alpha \beta}$ are Cauchy's measures of finite relative strain.

Consider next the three vectors $x^{i}{ }_{, \alpha} \underset{\mathfrak{a}}{D^{\alpha}}$, where, as before, the $D_{\mathfrak{a}}^{\alpha}$ are the material components of the initial values (reference state values) of the directors; i.e., $\underset{\mathfrak{a}}{D^{i}}=X_{, \alpha}^{i} \underset{\mathfrak{a}}{D^{\alpha}}$. The equation

$$
\underset{\mathfrak{a}}{d^{i}-x^{i}, \alpha} \underset{\mathfrak{a}}{D^{\alpha}}=0
$$

holds if and only if each director field is "material"; i.e., if and only if each director deforms and rotates along with the continuum. Multiplying the lefthand side of (8.14) by $x^{i}, \alpha$, we obtain the quantities

$$
-\gamma_{\mathfrak{a}}=\Delta_{\mathfrak{\alpha}}-a_{\alpha \beta} D_{\mathfrak{a}}^{\beta}
$$

which vanish if and only if each director is material. It follows that the variables $\gamma_{\alpha}$ are measures of the relative strain of the "micromedium" and its surrounding "macro-medium". To first order terms in the displacement gradient $u_{i: j}$ and the differences $\left(\underset{\mathbf{a}}{d^{i}}-\underset{\mathbf{a}}{D^{i}}\right)$ we have

$$
-\underset{\mathfrak{a}}{\gamma_{i}}=\left(d_{\mathfrak{a}}-\underset{\mathfrak{a}}{D_{i}}\right)-u_{i: j} \underset{\mathbf{a}}{D^{j}}
$$

provided again that one chooses the $X^{i}(\xi)$ for material coordinates. 
If for each rectangular Cartesian coordinate system $X^{i}$ one chooses for the directors $\underset{\mathfrak{a}}{D^{i}}$, the three unit vectors along the coordinate axes, then $D_{\mathfrak{a}}^{i}=\underset{\mathfrak{a}}{\delta^{i}}$ and the infinitesimal relative micro-macro strain measures (8.16) have the form

where

$$
\gamma_{\mathrm{a} i}=-\psi_{\mathrm{a} i}+u_{i: \mathrm{a}}
$$

$$
\psi_{\mathrm{a} i}=d_{\mathbf{a}}-{\underset{\mathrm{a}}{i}}_{1}
$$

given by MindLin $(1964,1)$. Since a different choice is made for the initial values of the directors for each coordinate system, the labeling index " $\mathfrak{a}$ " becomes a tensor index as in (8.17), and, in MindLIN's linear theory, $\psi_{a i}$ is a tensor of rank two under orthogonal transformations of the spatial coordinate system.

Finally, the variables $\Delta_{\alpha \beta}$ vanish in the reference configuration provided the initial values of the directors are constant vector fields in $M$; hence, they remain small if the deformation of the directors is not too large and inhomogeneous. To first order terms in the displacement gradient and the differences $\psi_{a i}$ we have

$$
\Delta_{\mathfrak{a}}{ }_{i j}=\psi_{\mathfrak{a} i, j}=\chi_{\mathfrak{a} i j}
$$

provided the coordinate system $X^{i}$ is rectangular Cartesian and the initial values of the director fields are unit vectors along the coordinate axes, where the $\varkappa_{\alpha i j}$ are the infinitesimal "micro-strain" gradient measures introduced by MiNDIIN.

Now it is clear from the definitions (8.10) and (8.15) of the strain measures $e_{\alpha \beta}$ and $\underset{a}{\gamma_{\alpha}}$ that any admissible energy function may be expressed in the form

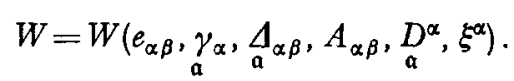

It should be emphasized that this function depends on the reference configuration through the $A_{\alpha \beta}$ and $D_{\mathfrak{a}}^{\alpha}$ because, for a given configuration $x_{t}(M)$, the values of the relative strain measures $e_{\alpha \beta}$ and $\underset{\mathfrak{a}}{\gamma_{\alpha}}$ depend on $A_{\alpha \beta}$ and $\underset{\mathfrak{a}}{D^{\alpha}}$.

\section{Cosserat Continua}

A Cosserat continuum is kinematically equivalent to the media with three deformable directors which we have been considering provided one adds the six independent constraints

$$
g_{i j} \underset{\mathfrak{a}}{d_{\mathfrak{b}}^{i}} \underset{\substack{d \\ \mathfrak{a} \mathfrak{b}}}{g}
$$

where $g$ is any constant, symmetric, nonsingular, positive definite matrix. We may obtain the Cosserats' equations of motion from the variational equation (5.4) which, in this case, must hold only for variations consistent with the six constraints (9.1). A variation is consistent with (9.1) if and only if

$$
\left.\stackrel{a}{d}_{(i} \delta d_{\mathfrak{a}}\right)=0 \text {, }
$$


where $\stackrel{\mathfrak{d}}{i}_{i} d_{\mathfrak{b}}^{i}=\stackrel{\mathfrak{d}}{\delta}_{\mathfrak{b}}^{\mathfrak{a}}$, so that the vectors $\stackrel{\mathfrak{a}}{d}_{i}$ are reciprocal to the set $d_{\mathfrak{a}}^{i}$. The equations of motion and boundary conditions for Cosserat media have the form

$$
\begin{gathered}
t_{i, j}^{j}+f_{i}=j^{-1} \dot{P}_{i}, \\
m_{i j, k}^{k}-t_{[i j]}+l_{[i j]}+P_{[i} \dot{x}_{j]}=j^{-1} \dot{Q}_{[i j]}, \\
t_{i}^{j} n_{j}=t_{i}, \\
m_{i j}{ }^{k} n_{k}=h_{[i j]},
\end{gathered}
$$

where all the quantities which appear have been defined in $\S 7$. These equations are the same as for a medium with deformable directors with the exception that Eq. (7.6) is replaced by its antisymmetric part and Eq. (7.8) is replaced by its antisymmetric part. One can easily perceive without a detailed derivation of these results how this reduction in the number of field equations and boundary conditions follows from the constraints (9.2).

Of particular interest to the history of the subject of couple-stresses in elasticity theories is the energy equation for a Cosserat continuum. It follows from the constraint equations (9.1) that the tensor,

$$
\widetilde{\omega}_{i j}={\stackrel{\mathfrak{d}}{d_{i}}}_{\mathfrak{a}_{j}},
$$

which measures the rate of rotation of a point in a Cosserat medium, is antisymmetric $\left(\widetilde{\omega}_{i j}=-\widetilde{\omega}_{i i}\right)$. It is possible, therefore, to write the energy equation (6.7) in the form

$$
\frac{d}{d t} \int_{P} E d \mathscr{F}=\int_{P}\left(f_{i} \dot{x}^{i}+\frac{1}{2} l_{[i j]} \widetilde{\omega}^{i j}\right) d \nu+\int_{P}\left(t_{i} \dot{x}^{i}+\frac{1}{2} h_{[i j]} \widetilde{\omega}^{i j}\right) d a .
$$

The rate of working of the extrinsic forces at the boundary of the part $P$ of the body appears here as the sum of the rate of working of the stress vector $t_{i}$ and a distribution of couples $h_{[i j]}$. This should be compared with the energy equation for a medium with deformable directors in which the rate of working contains additional terms representing the rate of working of a distribution $h_{(i j)}$ of self-equilibrated double forces. Perhaps the most important concept gained from a study of the mechanics of media with deformable directors is the relevance of such distributions of self-equilibrated tensions to the general theory of continuous media. We see that Eq. (9.8) does not hold generally in perfectly elastic media, but represents accurately the balance of energy only in certain special models.

\section{Nonsimple Elastic Materials}

The classical theory of elasticity can be generalized in quite another direction which also leads to a modification of the familiar concepts of stress. Rather than introducing rigid or deformable points, consider the following alternative line of thought. In the classical theory, the elastic energy of a body is given by an integral of the form

$$
E(M)=\int_{M} W d \mathscr{V}
$$


where $W$ is the energy per unit reference volume. For simplicity, in this section we shall consider only statics. Now the energy density $W$ at a point $\xi$ is determined by the instantaneous configuration $x_{t}(N)$ of an arbitrarily small neighborhood of points $N(\xi)$ containing the point $\xi$ under consideration. This principle of local action is basic to the classical theory, and to the generalization we wish to construct. Suppose that $x_{t}(\xi)$ has $p$ derivatives in $N(\xi)$. Then the relative position vector of the point $\xi$ and the point $\xi^{\prime} \in N(\xi)$ can be written in the form

$$
\begin{aligned}
x^{i}\left(\xi^{\prime}\right)-x^{i}(\xi)= & x^{i},{ }_{\alpha}(\xi) d \xi^{\alpha}+\frac{1}{2} x^{i},{ }_{\alpha \beta}(\xi) d \xi^{\alpha} d \xi^{\beta}+ \\
& +\ldots \frac{1}{p !} x^{i}, \alpha_{1} \alpha_{2} \ldots \alpha_{p}(\xi) d \xi^{\alpha_{1}} \ldots d \xi^{\alpha_{p}}+O\left(d^{p+1}\right)
\end{aligned}
$$

where $O\left(d^{p+1}\right)$ represents a term of order $p+1$ in the diameter $d$ of $N(\xi)$. We see that the possible configurations of the points $N(\xi)$ are classified more and more finely by the values of the successively higher position gradients at the point $\xi$. In the classical theory, the energy density has the form

$$
W=W\left(x^{i}, \alpha, \xi^{\alpha}\right),
$$

which means that all configurations of the particles $N(\xi)$ which correspond to the same values of the first order position gradient $x^{i}{ }_{\alpha}(\xi)$ are mapped into one and the same value of the energy density at $\xi$. An easy generalization of (10.3) consistent with the principle of local action is to assume that the energy density might also depend, however weakly, upon any finite number of higherorder position gradients. By such reasoning one is led to consider the mechanics of elastic media based on an expression for the elastic energy of the form

$$
E(M)=\int_{M} W\left(x^{i}, \alpha, x^{i}, \alpha \beta, \ldots, x^{i}, \alpha_{1} \alpha_{2} \ldots \alpha_{N}, \xi\right) d V .
$$

Following NolL's terminology $(1958,3)$, the classical theory based on $(10.3)$ is the theory of perfectly elastic simple materials. If any higher-order position gradient appears in (10.4), we call the corresponding material nonsimple. If $N$ is the order of the highest gradient actually present as an argument of the energy density, we call the material grade $N$. There follow some results for materials of grade 2. The general features of the theory of nonsimple materials are illustrated sufficiently well by materials of grade 2 , and the analysis of higher grade materials is only that much more complicated in details.

Let $P$ denote any part of a material of grade 2 . Its energy is given by

$$
E(P)=\int_{P} W\left(x^{i}, \alpha, x^{i}, \alpha \beta, \xi\right) d \mathscr{V} .
$$

The definition of the second-order position gradient is written out in full in Eq. (2.8). To obtain the equations of equilibrium and boundary conditions for the part $P$ of $M$ we assume the principle of virtual work

$$
\delta E(P)=\int_{P} F_{i} \delta x^{i} d \mathscr{V}+\int_{\partial P}\left(T_{i} \delta x^{i}+H_{i} D \delta x^{i}\right) d \mathscr{A}
$$

where $F_{i}$ is the body force per unit undeformed volume, $T_{i}$ are the tractions per unit undeformed area, $H_{i}$ are the hypertractions per unit undeformed area, 
and $D \delta x^{i}$ denotes the normal derivative of the variation $\delta x^{i}$ :

$$
D \delta x^{i}=\delta x^{i}{ }_{\alpha} N^{\alpha} .
$$

The variational equation (10.6) is postulated for arbitrary variations $\delta x^{i}\left(\xi^{\alpha}\right)$ of the position field. It can be shown that (10.6) is equivalent to the following system of equilibrium equations and boundary conditions:

$$
\begin{gathered}
T_{i, \alpha}^{\alpha}+F_{i}=0, \quad \xi \in P, \\
T_{i}^{\alpha} N_{\alpha}-D_{\alpha} H_{i}^{\alpha \beta} N_{\beta}+H_{i}^{\alpha \beta}\left(B_{\alpha \beta}-B^{\delta}{ }_{\delta} N_{\alpha} N_{\beta}\right)=T_{i}, \quad \xi \in \partial p, \\
H_{i}^{\alpha \beta} N_{\alpha} N_{\beta}=H_{i},
\end{gathered}
$$

where

$$
T_{i}^{\alpha}=\frac{\partial W}{\partial x^{i}, \alpha}-\left(\frac{\partial W}{\partial x^{i}, \alpha \beta}\right)_{, \beta}
$$

is a generalization of Kirchhoff's stress tensor of the classical theory, and

$$
H_{i}^{\alpha \beta}=\frac{\partial W}{\partial x^{i}, \alpha \beta}
$$

is the hyperstress tensor. In the boundary condition (10.9), $D_{\alpha} H_{i}^{\alpha \beta}$ is the surface gradient or surface divergence of the hyperstress defined by

$$
\begin{aligned}
H_{i}^{\alpha \beta}{ }_{, \alpha} & =D H_{i}^{\alpha \beta} N_{\alpha}+D_{\alpha} H_{i}^{\alpha \beta}, \\
D H_{i}^{\alpha \beta} & =H_{i}^{\alpha \beta}{ }_{, \delta} N^{\delta}
\end{aligned}
$$

and $B_{\alpha \beta}$ is the second fundamental form of the undeformed boundary of $P$ $\left(B_{b}^{\alpha}=A^{\alpha \delta} B_{\partial \beta}\right)$.

The equations of equilibrium and boundary conditions (10.8) and (10.9) for a material of grade 2 can be written in the following alternative spatial form:

where

$$
\begin{gathered}
t^{i j}{ }_{i j}+f^{i}=0, \quad \xi \in P, \\
t^{i j} n_{j}-d_{j} h^{j i k} n_{k}+h^{j i k}\left(b_{j k}-b_{q}^{q} n_{j} n_{k}\right)=t_{i}, \quad \xi \in \partial P, \\
h^{j i k} n_{j} n_{k}=h^{i},
\end{gathered}
$$

$$
t^{i j}=\sqrt{A / a} T^{i \alpha} x^{j}, \alpha
$$

is a generalization of Cauchy's stress tensor of the classical theory,

$$
h^{j i k}=\sqrt{A / a} H^{i \alpha \beta} x^{j}{ }_{, \alpha} x^{k}{ }_{, \beta}
$$

is the spatial form of the hyperstress tensor, and

$$
t^{i}=T^{i}(d \mathscr{A} / d a), \quad h^{i}=H^{i}(d \mathscr{A} / d a)
$$

are the tractions and hypertractions per unit deformed area, and $n_{i}$ is the unit outward normal to the deformed boundary of $P$. The tensor $b_{i j}$, which appears in the traction boundary condition $(10.14)_{1}$, is the second fundamental form of the deformed boundary, and $d_{j} h^{j i k}$ denotes the deformed surface divergence of $h^{i j k}$.

On substituting the expression (10.10) for the Kirchhoff tensor into the definition (10.15) of Cauchy's stress tensor and integrating certain of the terms 
by parts, we find the following relation:

$$
t^{i j}=\sqrt{A / a}\left[\frac{\partial W}{\partial x^{i}, \alpha} x_{, \alpha}^{j}+\frac{\partial W}{\partial x^{i}, \alpha \beta} x^{j}, \alpha \beta\right]-h^{j i k} .
$$

Now the expression in brackets in (10.18) is symmetric in the indices $i$ and $j$ if and only if the energy function is invariant under rigid rotations of the deformed body. It follows, therefore, that a necessary and sufficient condition that the energy be invariant under rigid rotations is that the CossERATs' moment equation

$$
t^{[i i]}+m^{j i k}=0
$$

be satisfied at each point $\xi$. Here the couple-stress $m^{i j k}$ is defined as the antisymmetric part

of the hyperstress.

$$
m^{i j k}=h^{[i j] k}
$$

If the energy density is invariant under all rigid transformations of the deformed body, then the variation of the elastic energy $E(P)$ of each part vanishes identically for every variation of the form

$$
x^{i}=a^{i}+b^{i j} x_{j}
$$

where $a^{i}$ and $b^{i j}=-b^{i i}$ are arbitrary constants and we assume that the spatial coordinate system (i) is rectangular Cartesian. It then follows directly from the variational equation (10.6) that no solution is possible unless the body force and the boundary data (tractions and hypertractions) satisfy jointly the compatibility conditions (conservation laws)

$$
\begin{gathered}
\int_{P} f_{i} d \psi+\int_{\partial P} t_{i} d a=0, \\
\int_{P} x_{[i} f_{j]} d v+\int_{\partial P}\left(x_{[i} t_{j]}+n_{[i} h_{j]}\right) d a=0 .
\end{gathered}
$$

It follows from these conditions that $\boldsymbol{n} \times \boldsymbol{h}$ is a couple per unit deformed area. The normal component $\boldsymbol{n} \cdot \boldsymbol{h}$ of the hypertraction enters neither of the compatibility conditions $(10.22)-(10.23)$. It represents a distribution of self-equilibrated forces.

By an argument similar to that given in detail in $\S 8$ it can be shown that every rotationally invariant energy density is expressible in the form

where

$$
W=W\left(e_{\alpha \beta}, q_{\alpha \beta \gamma}, A_{\alpha \beta}, \xi^{\alpha}\right)
$$

$$
q_{\alpha \beta \gamma}=x_{i, \alpha} x_{, \beta \gamma}^{i}
$$

and the $e_{\alpha \beta}$ are the finite relative strain measures defined in (8.10). The $q$ 's are expressible as linear combinations (an isomer) of the gradient of the strain measures $e_{\alpha \beta, \gamma}$ :

$$
q_{\alpha \beta \gamma}=e_{\alpha \beta, \gamma}+e_{\alpha \gamma, \beta}-e_{\gamma \beta, \alpha} .
$$

In words, therefore, what we have shown is that every admissible energy function of a material of grade 2 is expressible as a function of the six classical measures of finite strain, the material gradient of these measures, the initial metric components, and the material coordinates $\xi^{\alpha}(\xi)$. 


\section{Cosserat Materials with Constrained Rotations}

The particular class of elastic materials with couple-stresses considered by Grioli (1960, 2), Mindin \& Tiersten $(1962,2)$, and Toupin $(1962,1)$ is a subclass of the materials of grade 2 , the theory of which we have just outlined. In this section we shall show that this subclass may be viewed as consisting of Cosserat media in which the rotation of a point is constrained to equal the local rotation of the continuum. In $(1962,1)$ it was shown that this same class of materials comprised the materials of grade 2 for which the energy was a function

$$
W=W\left(e_{\alpha \beta}, e_{\alpha[\beta, \gamma]}, A_{\alpha \beta}, \xi^{\alpha}\right)
$$

independent of the completely symmetric part $e_{(\alpha \beta, \gamma)}$ of the strain gradient.

Now the directors of a Cosserat medium may be expressed in terms of their values in the reference configuration as follows,

$$
\underset{a}{d^{i}}(\xi, t)=\widetilde{R}_{\alpha}^{i}(\xi, t) \underset{\mathfrak{a}}{D^{\alpha}}(\xi)
$$

where the tensor $\widetilde{R}_{\alpha}^{i}$ satisfies the orthogonality condition

$$
g_{i j} \widetilde{R}_{\alpha}^{i} \widetilde{R}_{\beta}^{j}=A_{\alpha \beta} .
$$

Also, the position gradient $x^{i}, \alpha$ may be decomposed uniquely into a product

$$
x_{, \alpha}^{i}=R_{\beta}^{i}\left(a^{\frac{1}{2}}\right)_{\alpha}^{\beta}
$$

of an orthogonal tensor $R_{\alpha}^{i}$ satisfying (11.3) and the positive definite square $\operatorname{root}\left(a^{\frac{1}{2}}\right)_{\beta}^{\alpha}$ of $a_{\beta}^{\alpha}=A^{\alpha \gamma} a_{\gamma \beta}$. The tensor $R_{\alpha}^{i}$ measures the local rotation of the continuum. In a true Cosserat medium, the rotations $R_{\alpha}^{i}$ and $\widetilde{R}_{\alpha}^{i}$ are independent. Differentiating (11.2) we find that

$$
2 \widetilde{R}_{i} \dot{\widetilde{R}}_{j}=2 \dot{d}_{i}^{\mathfrak{a}} \dot{d}_{j}=\widetilde{\omega}_{i j}
$$

where $\widetilde{\omega}_{i j}$ are the rates of rotation which appear in the energy equation (9.8). Differentiating (11.4) we find that

$$
\dot{x}_{i, j}=\dot{R}_{i \alpha} R_{j}^{\alpha}+\xi^{\alpha}{ }_{, i} \xi^{\beta}, i\left(a^{-\frac{1}{2}}\right)_{\alpha}^{\gamma}\left(a^{\frac{1}{2}}\right)_{\gamma \beta} .
$$

The last term on the right-hand side of (11.6) is symmetric in $i$ and $j$, so that the classical vorticity or spin tensor of the continuum is given by

$$
\omega_{i j}=2 \dot{x}_{[j, i]}=2 R_{i}^{\alpha} \dot{R}_{j \alpha} .
$$

Suppose the rotation of the Cosserat triad $\underset{\mathfrak{a}}{d^{i}}$ is constrained to follow the local rotation of the continuum in the sense that we require

$$
R_{\alpha}^{i}=\tilde{R}_{\alpha}^{i}
$$

It then follows from (11.5) and (11.7) that the corresponding measures of the rate of rotation will be equal:

$$
\omega_{i j}=\widetilde{\omega}_{i j} .
$$


It now follows that, in a Cosserat medium with constrained rotations (constraints (11.8)), the energy equation has the form

$$
\frac{d}{d t} \int_{P} E d \mathscr{V}=\int_{P}\left(f_{i} \dot{x}^{i}+\frac{1}{2} l_{[i j]} \omega^{i j}\right) d v^{*}+\int_{P}\left(t_{i}^{k} \dot{x}^{i}+\frac{1}{2} m_{i j}{ }^{k} \omega^{i j}\right) n_{k} d a,
$$

where $\omega^{i j}$ is the ordinary vorticity.

The energy equation (11.10) was taken as a starting point in the special theory of grade 2 materials considered in $(1962,1)$ and $(1962,2)$. To the postulate (11.10) was added the assumption that the energy density $E$ had the special form

$$
E=\frac{1}{2} \varrho \dot{x}^{2}+W\left(x^{i}, \alpha, x^{i}, \alpha \beta, \xi^{\alpha}\right)
$$

which, because it lacks any contribution to the kinetic energy from the motion of the directors, is less general than that for the most complex type of Cosserat medium with constrained rotations. The spin density vanishes identically if the energy has form (11.11) but does not vanish for all Cosserat media with constrained rotations. It was found that the couple-stress for these materials was given by

$$
\begin{gathered}
m^{i(j k)}=2 \frac{\partial W}{\partial x^{j}, \alpha \beta} x_{, \alpha}^{i} x^{k}, \beta, \\
m^{[i j k]} \text { indeterminate. }
\end{gathered}
$$

Since $m^{i j k}=-m^{i i k}, m^{(i(j k))}=0$ so that one cannot have the relation (11.12) unless the energy function $W$ satisfies the ten independent differential equations

$$
\frac{\partial W}{\partial x^{(i}, \alpha \beta} x_{, \alpha}^{j} x_{\beta}^{k)}=0 \text {. }
$$

Rotational invariance of the energy requires that $W$ satisfy the three independent equations

$$
\frac{\partial W}{\partial x^{[i}, \alpha} x_{j], \alpha}+\frac{\partial W}{\partial x^{[i}, \alpha \beta} x_{j], \alpha \beta}=0 .
$$

The general solution of the equations (11.13) and (11.14) is an arbitrary function

$$
W=W\left(e_{\alpha \beta}, e_{\alpha[\beta, \gamma]}, \xi^{\alpha}\right) .
$$

This result shows that the Cosserat media with constrained rotations are a proper subclass of the materials of grade 2. My present feeling is that this special class of grade 2 materials does not warrant further special study or attention. The conditions (11.13) seem unnatural in view of the general theory of grade 2 materials; moreover, we know from the analysis of special problems that these materials have some peculiar properties. For example, it was found that the longitudinal waves in an isotropic material had a speed independent of their frequency as in the classical theory, but that shear waves were dispersive. Also, the deformation of the boundary of certain crystals caused by initial stress and hyperstress in a general material of grade 2 does not occur when expected in a Cosserat medium with contrained rotations. 


\section{Initial Stress and Hyperstress}

In the classical theory of elasticity a homogeneous initial stress in a homogeneous material can be transformed away by a suitable homogeneous transformation of the reference configuration. The stress-free configurations of a material in the classical theory are called natural states. In the classical theory, the existence of a natural state is the rule rather than the exception; for materials of grade 2, we shall show that the existence of a state in which both the stress and hyperstress vanish is the exception rather than the rule.

It was shown in $\S 10$ that the energy function of a grade 2 material was expressible as a function

$$
W=W\left(e_{\alpha \beta}, q_{\alpha \beta \gamma}, A_{\alpha \beta}, \xi^{\alpha}\right)
$$

of the strain measures $e_{\alpha \beta}$ and the linear combinations $q_{\alpha \beta \gamma}$ of the strain gradient. The function $W$ depends on the reference configuration $x_{T}(M)$, the material coordinate system $(\alpha)$, and the spatial coordinate system (i).

Using the formulas (10.10), (10.11), (10.15), and (10.16), we obtain the following expressions for the stress and hyperstress in terms of the function $W$ in (12.1):

$$
\begin{aligned}
t^{i j} & =\sqrt{(A / a)}\left[T^{\alpha \beta} x^{i}{ }_{, \alpha} x^{j}{ }_{, \beta}+2 H^{[\alpha \gamma] \beta} x^{i}{ }_{, \alpha \beta} x^{j},{ }_{, \gamma}-H_{, \beta}^{\gamma \alpha \beta} x_{, \gamma}^{i} x_{, \alpha}^{j}\right], \\
h^{i j k} & =\sqrt{(A / a)} H^{\alpha \beta \gamma} x^{i},{ }_{, \alpha} x^{j},{ }_{, \alpha} x^{k},{ }_{, \gamma},
\end{aligned}
$$

where

$$
T^{\alpha \beta}=\frac{\partial W}{\partial e_{\alpha \beta}}, \quad H^{\alpha \beta \gamma}=\frac{\partial W}{\partial q_{\alpha \beta \gamma}} .
$$

Now every property of a given material is fixed once and for all by its energy function $W$. We proceed now to lay down definitions of special materials in terms of properties of the function $W$.

If the reference configuration is changed from $X^{i}$ to $X^{\prime i}$, the variables upon which $W$ depends undergo the transformations

$$
\begin{aligned}
e_{\alpha \beta}^{\prime} & =e_{\alpha \beta}+\frac{1}{2}\left(A_{\alpha \beta}-A_{\alpha \beta}^{\prime}\right), \\
q_{\alpha \beta \gamma}^{\prime} & =q_{\alpha \beta \gamma}+\left(\left\{\begin{array}{c}
\delta \\
\alpha \beta
\end{array}\right\}_{A}-\left\{\begin{array}{c}
\delta \\
\alpha \beta
\end{array}\right\}_{A^{\prime}}\right) a_{\gamma \delta},
\end{aligned}
$$

and the energy function $W^{\prime}$ based upon this new choice for the reference configuration is defined in terms of $W$ by the relation

$$
W^{\prime}\left(e_{\alpha \beta}^{\prime}, q_{\alpha \beta \gamma}^{\prime}, A_{\alpha \beta}^{\prime}, \xi^{\alpha}\right)=\sqrt{\left(A / A^{\prime}\right)} W\left(e_{\alpha \beta}, q_{\alpha \beta \gamma}, A_{\alpha \beta}, \xi^{\alpha}\right) .
$$

We now define a homogeneous material as one for which a reference configuration exists such that, in a rectilinear material coordinate system for that configuration (i.e., a coordinate system such that $A_{\alpha \beta}=$ constant), the energy function does not depend on the point $\xi$. It is easy to see from the transformation laws (12.4) that if one such configuration exists, then any homogeneous deformation and/or translation

$$
X^{\prime i}=L_{j}^{i} X^{i}+D^{i}
$$

(we assume the spatial coordinate system (i) is rectilinear) of the reference configuration yields another reference configuration with the same properties. We call such a reference configuration of a homogeneous material undistorted. The 
general formalism does not require that the reference configuration be undistorted, but from this point on, let this be assumed. Also, let it be assumed that the material coordinate system is rectilinear.

From the definitions (12.2), the stress and hyperstress in an undistorted reference configuration of a homogeneous material are given by the simpler formulas

$$
\begin{aligned}
{ }_{0} t^{i j} & ={ }_{0} T^{\alpha \beta} X^{i}{ }_{, \alpha} X_{, \beta}^{i}, \\
{ }_{0} h^{i j k} & ={ }_{0} H^{\alpha \beta \gamma} X^{i}{ }_{, \alpha} X^{j}{ }_{, \beta} X^{k},{ }_{,} .
\end{aligned}
$$

The coefficients ${ }_{0} T^{\alpha \beta}$ and ${ }_{0} H^{\alpha \beta \gamma}$ and the initial values of the position gradient $X_{, \alpha}{ }_{, \alpha}$ are all constants provided $(\alpha)$ and (i) are rectilinear coordinate systems. The ${ }_{0} T^{\alpha \beta}$ and ${ }_{0} H^{\alpha \beta \gamma}$ are defined in terms of the energy function by

$$
\begin{gathered}
{ }_{0} T^{\alpha \beta}=\left.\frac{\partial W}{\partial e_{\alpha \beta}}\right|_{\boldsymbol{e}=\mathbf{q}=\mathbf{0}}, \\
{ }_{0} H^{\alpha \beta \gamma}=\left.\frac{\partial W}{\partial q_{\alpha \beta \gamma}}\right|_{\boldsymbol{e}=\mathbf{q}=0} .
\end{gathered}
$$

In general, it cannot be expected that for an arbitrary choice of the reference configuration these values of the "initial" stress and hyperstress will vanish. From the definition of $W^{\prime}$ in terms of $W$ given in (12.5) we can deduce how the stress and hyperstress in two reference configurations must be related.

where

$$
\begin{aligned}
& { }_{0} T^{\prime \alpha \beta}=\left.\frac{\partial W^{\prime}}{\partial e_{\alpha \beta}^{\prime}}\right|_{\boldsymbol{e}^{\prime}=\boldsymbol{\alpha}^{\prime}=0}=\left.\sqrt{\left(\mid A A^{\prime}\right)} \frac{\partial W}{\partial e_{\alpha \beta}}\right|_{\mathbf{e}=\left(\boldsymbol{A}^{\prime}-\boldsymbol{A}\right), \boldsymbol{q}=\mathbf{0}} \\
& =\sqrt{\left(A / A^{\prime}\right)}\left[{ }_{0} T^{\alpha \beta}+\left(\left.\int_{\varepsilon=0}^{1} \frac{\partial^{2} W}{\partial e_{\alpha \beta} e_{\gamma \delta}}\right|_{\boldsymbol{\alpha}=0} d \varepsilon\right)\left(A_{\gamma \delta}^{\prime}-A_{\gamma \delta}\right)\right] \\
& =\sqrt{\left(A / A^{\prime}\right)}\left[{ }_{0} T^{\alpha \beta}+C_{(\mathrm{av})}^{\alpha \beta \delta}\left(A_{\gamma \delta}^{\prime}-A_{\gamma}\right)\right] \text {, }
\end{aligned}
$$

The coefficient $C_{(\text {av. }}^{\alpha \beta \gamma \delta}$ is a sort of average elastic modulus of the material along a straight line in the space of strain measures $e_{\alpha \beta}$ joining the two points which correspond to the pair of reference configurations. Suppose that ${ }_{0} T^{\alpha \beta} \neq 0$ and we seek to determine the existence of a reference configuration such that ${ }_{0} T^{\prime \alpha \beta}=0$. From (12.9) we see that sufficient conditions for the existence of a transformation to such a new reference configuration are that $C_{(\mathrm{av} .)}^{\alpha \beta \delta}$ be nonsingular in the sense that $C_{(\mathrm{av} .)}^{\alpha \beta \delta} v_{\alpha \beta} v_{\gamma \delta}>0, v_{\alpha \beta} \neq 0$ and that

be positive definite.

$$
A_{\alpha \beta}^{\prime}=A_{\alpha \beta}-\left(C_{(\mathrm{av} .)}^{-1}\right)_{\alpha \beta \gamma \delta 0} T^{\gamma \delta}
$$

A similar analysis for the hyperstress yields the formula

$$
{ }_{0} H^{\alpha \alpha \beta \gamma}=\sqrt{\left(A / A^{\prime}\right)}\left[{ }_{0} H^{\alpha \beta \gamma}+D_{(\mathrm{av} .)}^{\alpha \beta \gamma \delta}\left(A_{\delta_{\varepsilon}}^{\prime}-A_{\delta \varepsilon}\right)\right] .
$$

Since the condition ${ }_{0} T^{\alpha \beta \beta}=0$ fixes the metric $A_{\alpha,}^{\prime}$, this condition determines the reference configuration to within a rigid motion. To require that the initial hyperstress ${ }_{0} H^{\prime \alpha \beta \gamma}$ vanish simultaneously leads, in general, to an overdetermination of the reference configuration. To sum up, we have the following theorem on initial hyperstress: 
For almost all uniform homogeneous materials of grade 2 there exists a reference configuration free of initial stress, but, except for special materials, such a natural state possesses initial hyperstress. The natural state is unique to within a rigid motion.

\section{Material Symmetry}

In each of the models of an elastic continuum which have been considered, the action density determines a material symmetry group. We shall illustrate the idea and definition of material symmetry using materials of grade 2 and restrict attention to statics so that it suffices to consider the energy density $W$. For these materials we are given a function

$$
W=W\left(e_{\alpha \beta}, q_{\alpha \beta \gamma}, A_{\alpha \beta}, \xi^{\alpha}\right) .
$$

Since $A_{\alpha \beta}$ is a Euclidean metric field, the material coordinates $(\alpha)$ can always be chosen in such a way that the components $A_{\alpha \beta}$ are constants

$$
A_{\alpha \beta}=\delta_{\alpha \beta} .
$$

Consider, then, the length-preserving transformations

$$
(L, D): M \rightarrow M
$$

of the material manifold into itself. Each such transformation is represented in a coordinate system $(\alpha)$ where (13.2) holds by a formula

$$
\xi^{\prime \alpha}=L_{\beta}^{\alpha} \xi^{\beta}+D^{\alpha}
$$

where $L$ is an orthogonal matrix so that $L L^{T}=1$.

The arguments in the energy function (13.1) transform as follows under each transformation (13.4) of the manifold:

$$
\begin{aligned}
e_{\alpha \beta}^{\prime} & =L_{\alpha}^{\gamma} L_{\beta}^{\delta} e_{\gamma \delta}, \\
q_{\alpha \beta \delta}^{\prime} & =L_{\alpha}^{\delta} L_{\beta}^{\gamma} L_{\delta}^{\xi} q_{\varepsilon \gamma \xi} .
\end{aligned}
$$

The material symmetry group of the material with energy function (13.1) is the set of all transformations $(L, D)$ such that

$$
W\left(e_{\alpha \beta}^{\prime}, q_{\alpha \beta \gamma}^{\prime}, \xi^{\prime \alpha}\right)=\sqrt{A / A^{\prime}} W\left(e_{\alpha \beta}, q_{\alpha \beta \gamma}, \xi^{\alpha}\right)
$$

throughout the domain of $W$.

If a group of transformations (13.3) is given for a material, the functional equation (13.6) may be viewed as a system of restrictions on the energy function for that material. The material symmetry group of a homogeneous, isotropic material is the set of all distance-preserving transformations (13.3). Crystals are characterized by crystallographic space groups (13.3), etc.

\section{The Boundary-Layer Effect in Crystals}

Low-energy electron-diffraction studies (cf., e.g., $(1961,1)$ ) of very clean surfaces of crystals have revealed that the spacing between the first few layers of atoms adjacent to a free surface differs a small amount from the uniform spacing of the deep layers. As an application of the theory of elastic materials 
of grade 2, we shall show how an initial hyperstress causes a deformation of a boundary-layer near a free surface in qualitative agreement with what has been observed.

For simplicity, let the material be homogeneous so that

$$
W=W\left(e_{\alpha \beta}, q_{\alpha \beta \gamma}\right) .
$$

We can define a natural length for the material with energy function (14.1) by the formula

where

$$
l^{2}=\frac{|F|}{|C|}
$$

$$
F^{\alpha \beta \gamma \delta \mu \xi}=\left.\frac{\partial^{2} W}{\partial q_{\alpha \beta \gamma} \partial q_{\delta \mu \xi}}\right|_{\boldsymbol{e}=\boldsymbol{q}=\mathbf{0}}, \quad C^{\alpha \beta \gamma \delta}=\left.\frac{\partial^{2} W}{\partial e_{\alpha \beta} \partial e_{\gamma \delta}}\right|_{\boldsymbol{e}=\boldsymbol{q}=\mathbf{0}}
$$

and the norm $|T|$ of any material tensor such as $F$ or $C$ is defined by

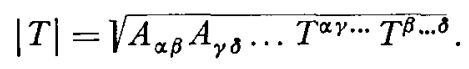

For simplicity and definiteness, consider now an infinite slab of a material of grade 2 which has plane parallel faces free of all tractions and hypertractions. Let its thickness be $2 L$ in its natural state. Let material coordinates coincide with the initial positions of the particles in a rectangular Cartesian coordinate system (i) chosen in such a way that the free surfaces of the slab correspond to the coordinate planes $|X|=\left|X^{1}\right|=L$.

We seek a solution of the equations of equilibrium (10.8) and (10.9) with vanishing body force, surface tractions, and hypertractions and which has the form

$$
\begin{aligned}
& x^{1}=x^{1}(X), \\
& x^{2}=X^{2}+u^{2}(X), \\
& x^{3}=X^{3}+u^{3}(X) .
\end{aligned}
$$

For a solution of this form, the position gradient $x^{i},{ }_{\alpha}$ is a function of $X$ only, Hence, the Kirchhoff tensor $T^{i \alpha}$ is a function of $X^{\prime \alpha}$ only, and the equilibrium equations have the simple form

$$
T_{, \alpha}^{i \alpha}=T_{, 1}^{i 1}=0 \text {. }
$$

Since the boundary of the slab is everywhere free of curvature, and the surface gradient of the hyperstress vanishes for solutions of the form (14.5), the boundary conditions simplify to

$$
\begin{aligned}
T^{i \alpha} N_{\alpha} & =T^{i 1}=0, \\
H^{i \alpha \beta} N_{\alpha} N_{\beta} & =H^{i 11}=0 \text { at }|X|=L .
\end{aligned}
$$

The general solution of (14.6) is $T^{i 1}=$ Constant, and from the boundary condition $(14.7)_{1}$ it follows that the constant is zero. Hence, the problem reduces to finding a solution of the lower-order equations

$$
T^{i 1}=0
$$

which satisfies the boundary conditions $(14.7)_{2}$. To simplify the notation, let

$$
y^{i}(X)=x^{i}, 1(X)
$$


and let a superposed dot denote differentiation with respect to $X=X^{1}$. Thus,

$$
\dot{y}^{i}=x^{i}, 11 \text {. }
$$

For deformations of the class (14.5), the energy is a certain function

$$
W=U\left(y^{i}, \dot{y}^{i}\right) \text {. }
$$

Expressed in terms of the function $U$, the equations (14.8) are

$$
\frac{\partial U}{\partial y^{i}}-\frac{\dot{\partial U}}{\partial \dot{y}^{i}}=0, \quad|X|<L,
$$

and the boundary conditions $(14.7)_{2}$ are

$$
\frac{\partial U}{\partial \dot{y}^{i}}=0, \quad|X|=L \text {. }
$$

It is interesting to note that, if one replaces the independent variable $X$ by the time $t$, the two-point boundary-value problem (14.12)-(14.13) is mathematically equivalent to the determination of the motions of a mass point in a force field $f_{i}=\partial U / \partial y^{i}$ such that the initial momentum $p_{i}=\partial U / \partial \dot{y}^{i}$ and the final momentum at some later time vanish. Systems of equations of this standard Eulerian form with one independent variable have an extensive literature. It appears, although I have not found a precise statement of the result, that sufficient conditions for the existence of a solution to the boundary-value problem (14.12)-(14.13) are the positive definiteness of the Hessian matrix

$$
l^{2} \alpha_{i j}=\frac{\partial^{2} U}{\partial \dot{y}^{i} \partial \dot{y}^{j}},
$$

in a suitable region of the phase space.

To exhibit the boundary-layer effect in more explicit form, let us write the variables $y^{i}$ as

$$
y^{i}=\delta_{1}^{i}+w^{i}(X) .
$$

The new variables $w e^{i}(X)$ all vanish when the particles are in the natural, reference configuration. If the equilibrium configuration of the slab differs from the reference configuration by a motion with small strain and small rotation, then the dimensionless variables $w^{i}$ are all small:

$$
\left|w^{i}\right| \ll 1 \text {. }
$$

If it also be assumed that the gradient of the relative deformation and rotation is small in the sense that

$$
l\left|\dot{w}^{i}\right| \ll 1,
$$

then the solution for $w^{i}$ is approximated very closely by the solution of the linearized equations of equilibrium

$$
l^{2} \alpha_{i j} \ddot{w}^{j}+l \beta_{[i j]} \dot{w}^{j}-\gamma_{i j} w^{j}=0,
$$

which satisfies the linearized boundary conditions

$$
l \alpha_{i j} \dot{w}^{j}+\beta_{i j} w^{j}=-\mu_{i}, \quad|X|=L
$$


where $\alpha_{i j}$ is the dimensionless constant tensor obtained by setting $w^{i}=0$ in the right-hand side of (14.14), and the dimensionless tensors $\beta_{i j}, \gamma_{i j}$, and $\mu_{i}$ are defined by

$$
\begin{gathered}
l \beta_{i j}=\left.\frac{\partial^{2} U}{\partial \dot{y}^{i} \partial y^{j}}\right|_{\boldsymbol{w}=0}, \\
\gamma_{i j}=\left.\frac{\partial^{2} U}{\partial y^{i} \partial y^{j}}\right|_{\boldsymbol{w}=\mathbf{0}}, \\
l \mu_{i}=\left.\frac{\partial U}{\partial \dot{y}^{i}}\right|_{\boldsymbol{w}=0} .
\end{gathered}
$$

Note that $\mu_{i}$ measures the initial hypertraction on planes normal to the $X$ axis. The $\gamma_{i j}$ are the elastic constants $C_{i 1 j 1}$ of the classical theory of elasticity.

To solve the boundary-value problem (14.18)-(14.19), set

$$
w^{i}=W^{i} e^{k X / l}
$$

where the $W^{i}$ are constants. Substituting (14.21) into the equilibrium equations (14.18), one gets the linear homogeneous system

$$
\left(k^{2} \alpha_{i j}+k \beta_{[i j]}-\gamma_{i j}\right) W^{j}=0
$$

which has a nonzero solution if and only if $k$ is a root of the characteristic equation

$$
P(k)=\operatorname{det}\left\|k^{2} \alpha_{i j}+k \beta_{[i j]}-\gamma_{i j}\right\|=0 .
$$

It follows from the symmetry of the coefficients $\alpha_{i j}$ and $\gamma_{i j}$ and the antisymmetry of the coefficients $\beta_{[i j]}$ that the sixth degree polynomial $P(k)$ is even in $k$ : $P(k)=P(-k)$. Hence, $P(k)$ is a bicubic, and if $k$ is a root of (14.23), so also is $-k$. Let us assume as a condition on the strain energy function that $\alpha_{i j}$ and $\gamma_{i j}$ are positive definite ${ }^{\star}$. Since $P(k)$ is bicubic, it is not difficult to determine the conditions on the coefficients $\alpha_{i j}, \beta_{i j}$, and $\gamma_{i j}$ such that all of the roots of (14.23) are real. With positive definite $\alpha_{i j}$ and $\gamma_{i j}$, the roots are all real for sufficiently small $\beta_{i j}$. We shall assume that all six roots $k= \pm k, a=1,2,3$ are real. Then the general solution of the equilibrium equations can be written in the form

$$
w^{i}=\sum_{a}\left\{A_{a}^{A} \frac{\cosh \left({ }_{a} X / l\right)}{\cosh (k L / l)} U_{a}^{i}+\underset{a}{B} \frac{\sinh (\underset{a}{a} X / l)}{\sinh \left({ }_{a} L / l\right)} V_{a}^{i}\right\}
$$

where $U_{a}^{i}$ and $V_{a}^{i}$ are unit vectors. Substituting (14.24) into the boundary conditions (14.19), one obtains a system of six linear equations for the six scalar amplitudes $\underset{a}{A}, \underset{a}{B}, a=1,2,3$. These always have a solution which is linear in the initial hyperstress $\mu_{i}$. The amplitudes have been so defined that the coefficients in the linear relation are of order unity. Thus, when the thickness of the slab $L$ is much greater than the characteristic length $l$ of the material, one sees by inspection of the solution (14.24) that the strain and rotation determined by $w^{i}$ decays exponentially to zero from values on the surface of the slab which are linear functions of the initial hyperstress.

* MindLiN $(1962,2)$ has shown that, in the linear theory of materials of grade 2 , uniqueness of the usual boundary-value problems holds under these conditions and fails, in general, if they are not satisfied. 
Acknowledgments. I wish to thank Professor MINDLIN for his comments on the manuscript and for the many discussions we have had over the last two years concerning elasticity theory in general, and non-simple elastic materials in particular. The main ideas of his treatment of elastic materials with micro-structure were revealed to me in these discussions as early as June 1962, and I was in possession of a complete draft of his paper $(1963,2)$ in September 1963.

I am especially grateful to Professor TRUesdell for his specific comments on the manuscript and more deeply for his constant encouragement and guidance.

\section{References}

1850 1. CAUChy, A. L., Mémoire sur les systèmes isotropes de points materiels. Mém. Acad. Sci. 22, p. $615=$ Oeuvres (1) 2, 351.

1909 1. Cosserat, E., \& F., Théorie des Corps Déformables. Paris: Hermann \& Fils.

1914 1. Hellinger, E., Die allgemeinen Ansätze der Mechanik der Kontinua. Enzy. d. Math. Wiss. IV, 4, p. 30.

2. Heun, K., Ansätze und allgemeine Methoden der Systemmechanik. Enzy. d. Math. Wiss. IV, 2, p. 11.

1918 1. KleIN, F., Nachr. kgl. Ges. Wiss. Göttingen, 235.

2. Noether, E., Nachr. kgl. Ges. Wiss. Göttingen, 171.

1929 1. Jaramillo, T. J., A Generalization of the Energy Function of Elasticity Theory. Dissertation, U. of Chicago.

1952 1. TRUesdell, C., The Mechanical Foundations of Elasticity and Fluid Dynamics. J. Rational Mech. and Anal. 1, 125.

1956 1. Tiffen, R., \& A. C. Stevenson, Elastic Isotropy with Body Force and Couple. Quart. J. Mech. and Applied Math. 9, 306.

1958 1. Ericksen, J. L., \& C. Truesdell, Exact Theory of Stress and Strain in Rods and Shells. Arch. Rational Mech. and Anal. 1, 295.

2. GÜNTHER, W., Zur Statik und Kinematik des Cosseratschen Kontinuums. Abh. Braunschweigische Wiss. Ges. 10, 195.

1960 1. Truesdell, C., \& R. Toupin, The Classical Field Theories. Encyclopedia of Physics, Vol. III/1. Berlin-Göttingen-Heidelberg: Springer.

2. Grioli, G., Elasticità asimmetrica. Ann. di Mat. pura ed appl., Ser. IV, 50, 389.

1961 1. Germer, L. H., A. U. MacRae, \& C. D. Hartman, J. Appl. Phys. 32, 2432.

1962 1. Toupin, R., Elastic Materials with Couple-stresses. Arch. Rational Mech. Anal. 11, 385.

2. Mindlin, R. D., \& H. F. Tiersten, Effects of Couple-stresses in Linear Elasticity. Arch. Rational Mech. Anal. 11, 415.

3. Schaefer, H., Versuch einer Elastizitätstheorie des zweidimensionalen ebenen Cosserat-Kontinuums, Miszellaneen der Angewandten Mechanik. Festschrift W. Tollmien. Berlin: Akademie-Verlag.

1963 1. Toupin, R., \& D. Gazis, Surface Effects and Initial Stress in Continuum and Lattice Models of Elastic Crystals. To appear in the Proceedings of the First International Congress on Lattice Dynamics held at Copenhagen, 1963.

2. MINDlin, R. D., Microstructure in Linear Elasticity. Technical Report No. 50, ONR Project NR 064-388, Columbia Univ. = Arch. Rational Mech. Anal. 16, 51-78 (1964).

3. Toupin, R. A., Equivalence of the principles of conservation and invariance in non-simple elastic materials. Lecture presented at the first meeting of the Society for Natural Philosophy held at The Johns Hopkins University, Baltimore, March 1963.

IBM Watson Research Center Yorktown Heights, New York 